\title{
Unintended Consequences Following the 2014 American Academy of Pediatrics Policy Change for Palivizumab Prophylaxis among Infants Born at Less than 29 Weeks' Gestation
}

\author{
Mitchell Goldstein, MD ${ }^{1}$ Leonard R. Krilov, MD² Jaime Fergie, $\mathrm{MD}^{3}$ Lance Brannman, $\mathrm{PhD}^{4}$ \\ Sally W. Wade, $\mathrm{MPH}^{5}$ Amanda M. Kong, $\mathrm{DrPH}^{6} \quad$ Christopher S. Ambrose, MD, MBA ${ }^{4}$
}

${ }^{1}$ Division of Neonatology, Department of Pediatrics, Loma Linda University Children's Hospital, Loma Linda, California

2 Division of Pediatric Infectious Disease, Children's Medical Center, NYU Winthrop, Mineola, New York

${ }^{3}$ Department of Infectious Diseases, Driscoll Children's Hospital, Corpus Christi, Texas

${ }^{4}$ Biopharmaceutical Medical Department, AstraZeneca,

Gaithersburg, Maryland

${ }^{5}$ Wade Outcomes Research and Consulting, Salt Lake City, Utah

6 Life Sciences Department, IBM Watson Health, Cambridge, Massachusetts
Address for correspondence Mitchell Goldstein, MD, Division of Neonatology, Department of Pediatrics, Loma Linda University Children's Hospital, 11175 Campus Street, Suite \#11121, Loma Linda, CA 92354 (e-mail: MGoldstein@llu.edu).

Am J Perinatol 2021;38(suppl S1):e201-e206.

\section{Abstract \\ Keywords \\ - respiratory syncytial virus \\ - immunoprophylaxis \\ - hospitalization rates \\ - palivizumab \\ Objective The aim of this study is to compare outpatient respiratory syncytial virus (RSV) immunoprophylaxis (IP) use and relative RSV hospitalization (RSVH) rates for infants $<29$ weeks' gestational age (wGA) versus term infants before and after the 2014 American Academy of Pediatrics (AAP) policy change. \\ Study Design Infants were identified in the MarketScan Commercial and Multi-State Medicaid databases. Outpatient RSV IP receipt and relative $<29 \mathrm{wGA} /$ term hospitali- zation risks in 2012 to 2014 and 2014 to 2016 were assessed using rate ratios and a difference-in-difference model. \\ Results Outpatient RSV IP receipt by infants $<29$ wGA and aged $<3$ months in the Commercial and Medicaid populations and those aged 3 to $<6$ months in the Medicaid population declined after 2014. Relative RSVH risks for infants $<29$ wGA were numerically greater after 2014, with infants aged $<3$ months and Medicaid infants experiencing the greatest increases. Difference-in-difference results indicated a significantly increased relative risk of RSVH for infants $<29$ wGA versus term (both cohorts aged 0 to $<6$ months) in the Medicaid-insured population (1.68, $p=0.0054)$. A nonsignificant increase of similar magnitude occurred in the commercially insured population (1.57, $p=0.2867$ ). \\ Conclusion The 2014 policy change was associated with a decrease in RSV IP use and an increase in RSVH risk among otherwise healthy infants $<29$ wGA.}

received

August 6, 2019 accepted after revision

February 25, 2020

published online

April 16, 2020
DOI https://doi.org/

10.1055/s-0040-1709127.

ISSN 0735-1631.

\footnotetext{
(c) 2020. The Author(s).

This is an open access article published by Thieme under the terms of the Creative Commons Attribution-NonDerivative-NonCommercial-License, permitting copying and reproduction so long as the original work is given appropriate credit. Contents may not be used for commercial purposes, or adapted, remixed, transformed or built upon. (https://creativecommons.org/ licenses/by-nc-nd/4.0/) Thieme Medical Publishers, Inc., 333 Seventh Avenue, 18th Floor New York, NY 10001, USA
} 
Respiratory syncytial virus (RSV) is the leading cause of infant hospitalizations in the United States, and preterm infants are at increased risk. ${ }^{1-5}$ Palivizumab was approved in 1998 and is currently indicated to prevent severe serious lower respiratory tract infection caused by RSV in infants born at $\leq 35$ weeks' gestational age (wGA) who are aged $\leq 6$ months at the start of the RSV season. Palivizumab is also approved for infants with chronic lung disease of prematurity (CLDP, formerly referred to as bronchopulmonary dysplasia) or hemodynamically significant congenital heart disease (CHD) who are aged $\leq 24$ months at the start of the RSV season. ${ }^{6}$ In 2014, the American Academy of Pediatrics (AAP) stopped recommending RSV immunoprophylaxis (IP) with palivizumab for otherwise healthy infants without CLDP or CHD born at 29 to 34 wGA while continuing to recommend RSV IP for infants born at $<29 \mathrm{wGA} .^{7,8}$ The declines in RSV IP and concurrent increases in RSV hospitalizations (RSVH) among infants born at 29 to 34 wGA have been described, ${ }^{4,5,9}$ but it is unknown whether there have been unintended spillover effects on preterm infants $<29$ wGA. This study addresses this question by evaluating outpatient RSV IP receipt and RSVH rates prior to and following the policy change among infants $<29 \mathrm{wGA}$ relative to term infants in two large-scale databases of US health care insurance claims.

\section{Materials and Methods}

\section{Data Sources}

The analyses were conducted using the IBM Watson Health MarketScan Commercial Claims and Encounters (Commercial) and Medicaid Multi-State (Medicaid) databases. Both contain inpatient and outpatient medical claims, outpatient pharmacy claims, and enrollment information for enrollees. The Commercial population includes enrollees from selfinsured employers and commercial health plans. The Medicaid population includes enrollees from Medicaid insurance plans in several geographically diverse states. The data were previously collected and statistically deidentified, and are compliant with the conditions set forth in Sections 164.514 (a)-(b)(1)ii of the Health Insurance Portability and Accountability Act of 1996 Privacy Rule; therefore, approval from an institutional review board was not required.

\section{Patient Selection}

Infants born between July 1,2011 and June 30, 2016 were selected from the two databases using International Classification of Diseases, Ninth Revision, Clinical Modification (ICD-9-CM) and ICD-10-CM diagnosis codes and diagnosis-related group (DRG) codes on inpatient claims. Gestational age and conditions associated with increased risk for RSV were derived from diagnosis, DRG, procedure, and medication codes. Infants with the following conditions and GAs were excluded from the final study populations: chronic lung disease; CHD; cystic fibrosis; immunodeficiency; congenital anomalies of respiratory system; neuromuscular disease; organ transplants; other neuromuscular, immunological, or genetic conditions; preterm infants born 29 to $<37$ wGA; and term infants with major health problems. Otherwise healthy infants born at $<29$ wGA and term infants without major health problems contributed to the current analyses.

\section{Infant Follow-up}

Infants were followed from birth through the earliest of the following events: end of the first 6 months of life, death, or end of continuous enrollment in the database. Infants contributed person-time after birth hospitalization discharge and while aged $<6$ months during the RSV season (defined as November 1 through March 31 to reflect the average peak season across the United States). We focused on infants aged $<6$ months because RSV IP utilization and RSVH risks decrease after 6 months of age. ${ }^{4}$ Infant person-time was also stratified based on chronological age (CA) during the RSV season: $<3$ months and 3 to $<6$ months. The total number of days that an infant contributed during the RSV season was divided by 151 (the number of days during the stipulated 5-month average national RSV season) to calculate follow-up time in infant-seasons.

\section{Outcomes}

The primary outcomes of interest were receipt of outpatient RSV IP among infants $<29$ wGA and the relative risk of RSVH in infants $<29$ wGA compared with term infants. Results were compared for the combined RSV seasons from 2012 to 2014 versus those from 2014 to 2016. Because RSV coding on administrative claims is influenced by testing and coding practices, ${ }^{10-12}$ recorded RSV diagnoses do not capture all RSV disease. Therefore, the rates of all-cause bronchiolitis hospitalizations, which include disease due to RSV and other respiratory pathogens, ${ }^{13}$ were also compared between the two time periods to assess the broader potential effects of the AAP policy change and to provide insights into whether any changes in RSVH rates may have been due to changes in diagnosis coding on claims.

RSV IP was identified using outpatient medical and pharmacy claims with palivizumab-specific drug codes (National Drug Codes) or administration codes (Current Procedural Terminology or Healthcare Common Procedure Coding System). RSVH was identified by evaluating inpatient claims for an ICD9-CM $(079.6,466.11,480.1)$ or ICD-10-CM diagnosis (B974, $\mathrm{J} 205, \mathrm{~J} 121, \mathrm{~J} 210$ ) indicating RSV in any position on the claim. Allcause bronchiolitis hospitalizations are those with a diagnosis of either RSV bronchiolitis (ICD-9-CM diagnosis code $=466.11$ or ICD-10-CM diagnosis code $=\mathrm{J} 21.0$ in any position on a claim) or unspecified bronchiolitis (ICD-9-CM code $=466.19$ or ICD-10-CM code $=\mathrm{J} 211$, J218, or J219 in any position on a claim and no diagnoses of influenza, bacterial pneumonia, or other viral pathogen [e.g., human rhinovirus, SARS, and adenovirus] within 3 days of the unspecified bronchiolitis claim).

\section{Statistical Analyses}

For RSV IP utilization during the RSV season, the proportions of infants with $\geq 1$ outpatient claim for palivizumab during the 2012 to 2014 and 2014 to 2016 RSV seasons were calculated. Differences in these proportions between the two time periods were compared using Chi-squared tests.

Rate ratios ( $<29$ wGA/term) comparing RSVH and allcause bronchiolitis hospitalization rates for infants of the same CA were calculated to account for seasonal variations in RSV circulation and severity. Because absolute risks depend on an infant's chronological age, for each CA category we also 
compared hospitalization rates of infants $<29 \mathrm{wGA}$ relative to a common reference, term infants aged 0 to $<6$ months.

Multivariable difference-in-difference (DID) models that hold patient characteristics (gender and gestational age) constant across RSV seasons were estimated for all infants aged $<6$ months to compare rate ratios of $<29$ wGA versus term infants before and after the 2014 AAP policy change. Because the modeled outcomes (hospitalization events) were counts, we used generalized linear models with a Poisson error distribution, a log link, and a log offset term to represent exposure. The DID ratio is as follows:

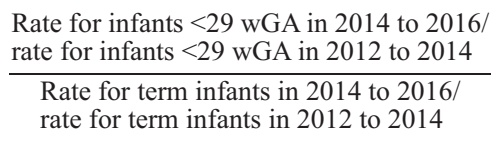

It is derived by exponentiating the $\beta 3$ coefficient in the patient-level regression specification:

Ln (number of hospitalizations/days of follow-up) = intercept $+\beta 1^{*}$ gestational_age $+\beta 2^{*} R S V \_$season $+\beta 3^{*}$ gestationa1_age*RSV_season $+\beta 4^{*}$ sex + error

$p$-values $<0.05$ were considered statistically significant.

\section{Results}

\section{Infant Characteristics}

There were 1,142,777 commercially insured infants and $1,458,060$ Medicaid-insured infants born and discharged alive from their birth hospitalization in the two databases from July 1, 2011 to June 30, 2016 (-Supplementary Table S1 [available in the online version]). After removing infants with CLDP, CHD, and rare and complex medical conditions from the Commercial sample, 4,701 infants born at $<29$ wGA $(0.7 \%$ of the final sample) and 668,619 healthy term infants (99.3\%) were identified for inclusion. The final Medicaid sample included 9,334 infants born at $<29$ wGA (1.0\%) and 908,594 healthy term infants $(99.0 \%)$.

Preterm infants $<29$ wGA aged $<6$ months in the 2012 to 2014 RSV seasons contributed 543 and 1,113 infant-seasons in the Commercial and Medicaid analyses, respectively, and 412 (Commercial) and 1,084 (Medicaid) infant seasons in the 2014 to 2016 period (- Supplementary Table S2 [available in the online version]). The number of infant-seasons contributed by commercially insured and Medicaid-insured term infants was 134,655 and 194,682 in the 2012 to 2014 period and 97,546 and 178,214 in the 2014 to 2016 period. The overall MarketScan database was smaller in the 2014 to 2017 period, resulting in lower infant frequencies. The mean birth hospitalization lengths of stay for infants $<29$ wGA in the 2012 to 2014 and 2014 to 2016 periods, respectively, were 73.4 and 76.2 days for commercially insured infants and 76.5 and 77.2 days for Medicaid-insured infants. Mean birth hospitalization lengths of stay for term infants over the same periods were 3.0 and 2.9 days (Commercial) and 3.1 and 3.1 (Medicaid). Demographic characteristics for infants who contributed person-time while $<6$ months CA during the 2012 to 2016 RSV seasons are in - Supplementary Table S3 (available in the online version).

\section{Outcomes}

\section{Respiratory Syncytial Virus Immunoprophylaxis}

Outpatient RSV IP receipt declined after 2014 for infants $<29$ wGA aged $<6$ months, with the greatest decline observed among infants $<3$ months CA during the season (-Table 1 ). Between the 2012 and 2014 and 2014 and 2016 RSV seasons, statistically significant decreases of 46 and $36 \%$ were observed for infants $<3$ months CA in the Commercial and Medicaid populations, respectively. Among infants 3 to $<6$ months CA, the $24 \%$ decrease in RSV IP observed in the Medicaid population achieved statistical significance; however, the 7\% RSV IP decrease in the Commercial population did not achieve statistical significance.

\section{Hospitalizations}

For each CA category and in each population, RSVH rate ratios comparing $<29 \mathrm{wGA}$ to term infants were numerically greater in the 2014 to 2016 RSV seasons compared with the 2012 to 2014 RSV seasons ( - Fig. 1), ranging from 0.5 to 5.0 in 2012 to 2014 and from 2.6 to 5.8 in 2014 to 2016 (-Supplementary Table $\$ 4$ [available in the online version]). The greatest proportional increases in preterm/term RSVH rate ratios occurred among infants $<3$ months CA ( $=$ Figs. 1 and 2A,B). Absolute RSVH rates for all groups can be found in - Supplementary Table S5 (available in the online version).

All-cause bronchiolitis hospitalization rates for infants $<29$ wGA relative to term infants were also numerically greater in 2014 to 2016 for infants 0 to $<3$ months CA in both populations and for those 3 to $<6$ months CA in the Medicaid population (- Supplementary Table S4 [available in the online version]). A numerically lower preterm/term all-cause

Table 1 Proportion of infants < 29 weeks' gestational age receiving outpatient respiratory syncytial virus immunoprophylaxis in 2012 to 2014 versus 2014 to 2016 respiratory syncytial virus seasons

\begin{tabular}{|lllllll}
\hline & Commercial & & & Medicaid & \\
& $\begin{array}{l}2012 \text { to } 2014 \\
\text { RSV seasons }\end{array}$ & $\begin{array}{l}\text { 2014 to } 2016 \\
\text { RSV seasons }\end{array}$ & \% Decline & $\begin{array}{l}\text { 2012 to } 2014 \\
\text { RSV seasons }\end{array}$ & $\begin{array}{l}\text { 2014 to 2016 } \\
\text { RSV seasons }\end{array}$ \\
$<3$ mo CA (\%) & 19.8 & 10.5 & $46^{\text {a }}$ & 14.6 & 9.3 & $36^{\text {a }}$ \\
3 to $<6$ mo CA (\%) & 46.4 & 43.3 & 7 & 43 & 32.8 & $24^{\text {a }}$ \\
\hline
\end{tabular}

Abbreviations: CA, chronological age; IP, immunoprophylaxis; RSV, respiratory syncytial virus; wGA, weeks' gestational age. ${ }^{\mathrm{a}} \mathrm{p}<0.05$. 


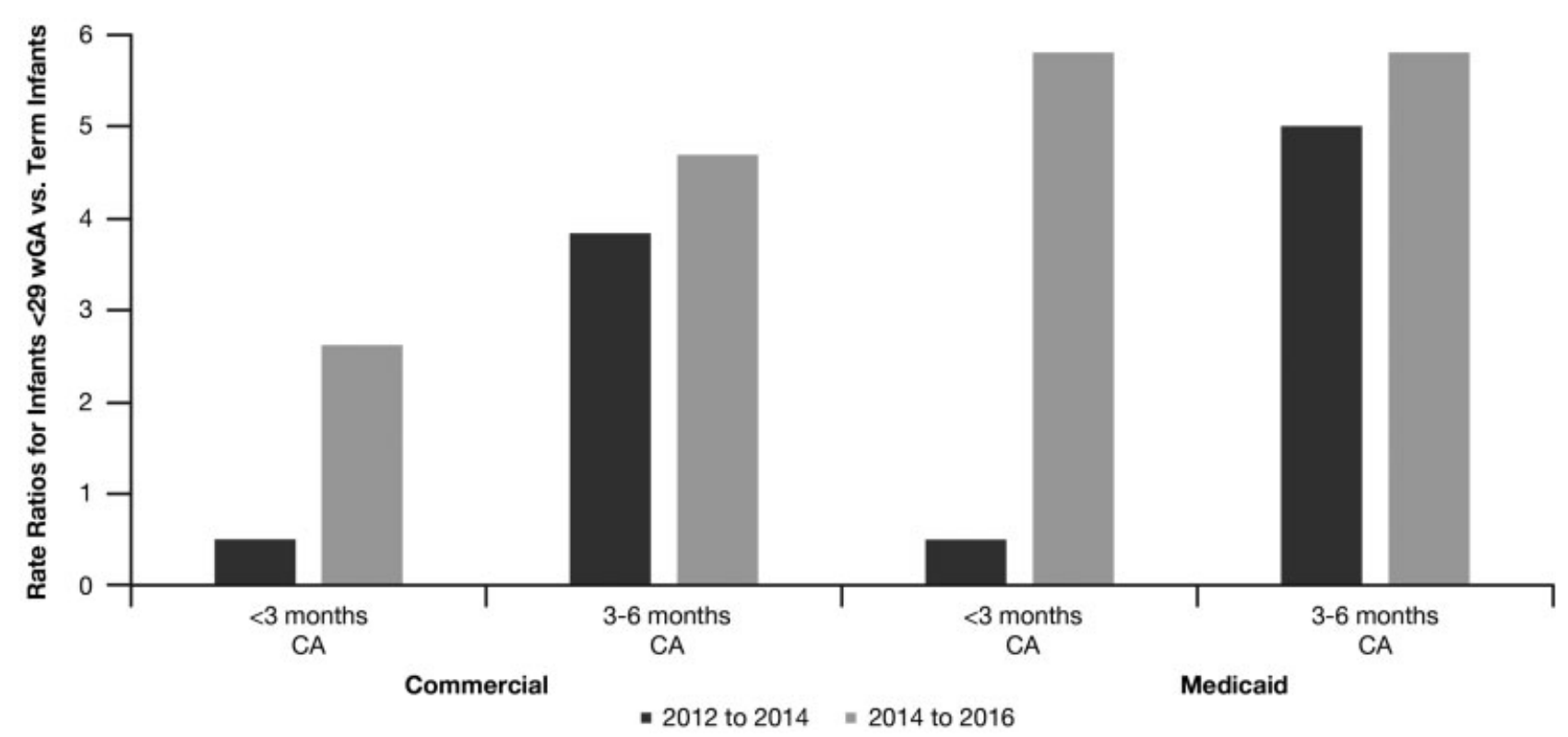

Fig. 1 RSV hospitalization rate ratios for infants $<29$ wGA versus term infants of the same chronological age during the 2012 to 2014 and 2014 to 2016 RSV seasons. CA, chronological age; RSV, respiratory syncytial virus; wGA, weeks' gestational age.

bronchiolitis hospitalization rate ratio was observed in 2014 to 2016 among infants $<29$ wGA aged 3 to $<6$ months in the Commercial population ( - Supplementary Table $\$ 4$ [available in the online version]). It is unlikely that the increase in RSVH rates observed in the postpolicy change period are due to changes in the way claims are coded because all-cause bronchiolitis hospitalization rates also were higher in the 2014 to 2016 period. Absolute all-cause bronchiolitis hospitalization rates for all groups can also be found in -Supplementary Table S5 [available in the online version].

Among Medicaid-insured infants $<6$ months $\mathrm{CA}$, the multivariable DID model results indicated that RSVH and all-cause bronchiolitis hospitalization risks associated with prematurity of $<29$ wGA compared with term births were $68 \%(p=0.0054)$ and $53 \%(p=0.0025)$ higher in the postpolicy change period compared with the prepolicy change period ( - Table 2). Among commercially insured infants $<6$ months CA, preterm/term hospitalization rate ratios were not statistically significant; however, numerical differences of a $57 \%$ increase in RSVH and a 36\% decrease in all-cause bronchiolitis hospitalizations in 2014 to 2016 compared with 2012 to 2014 were estimated from the models.

\section{Discussion}

Prior to 2014, the AAP policy recommended RSV IP for infants born at 29 to 34 wGA. After the 2014 AAP policy change, RSV IP was no longer recommended for infants born at 29 to 34 wGA. Previous analyses of the IBM Watson Health MarketScan databases that also examined the 2012 to 2014 and 2014 to 2016 RSV seasons demonstrated significant increases in the risk of RSVH during the 2014 to 2016 season for commercially and Medicaid-insured infants born at 29 to 34 wGA relative to term infants (Commercial: 2.00, $p<0.0001$, Medicaid: $1.46, p<0.0001){ }^{5}$
The results of the present analysis showing a decrease in RSV IP receipt and an increase in RSVH among infants born at $<29$ wGA are concerning because RSV IP continued to be recommended for infants $<29$ wGA following the 2014 AAP policy change. The observed decreases in RSV IP and relative increases in RSVH were greatest among infants $<3$ months CA and among those insured by Medicaid.

The 2014 policy change may have also contributed to a significant disparity of care between commercially insured infants and Medicaid-insured infants 3 to $<6$ months CA, as differences in levels of use observed prior to the change widened considerably after the change. Awareness of these unintended consequences is critical to developing a comprehensive understanding of the policy change's impact. ${ }^{14,15}$ The potential for spillover effects of clinical guidelines should be routinely considered and evaluated given the complex and multifactorial nature of health care delivery. ${ }^{9}$ Such effects have been noted in other settings, including gynecological screening and treatment of alcohol withdrawal. ${ }^{16,17}$

Limitations in the study may affect estimated values, the identification of outcomes, and statistical variability. First, no data were available regarding inpatient RSV IP receipt; therefore, RSV IP use among infants $<29$ wGA, who often remain hospitalized for at least several weeks after birth, is likely underestimated. Second, RSVH were identified using diagnosis codes from inpatient claims because confirmatory laboratory results were not available in the Commercial and Medicaid claims databases. Third, as noted above, inpatient utilization of palivizumab was not captured because inpatient doses, typically given at an infant's birth hospitalization discharge, are not recorded as separate line items on health care insurance claims. This lack of data on inpatient RSV IP receipt lowered our ability to detect an association between reported RSV IP receipt and RSV or bronchiolitis hospitalizations among infants $<3$ months $\mathrm{CA}$ and precluded directly testing associations between 

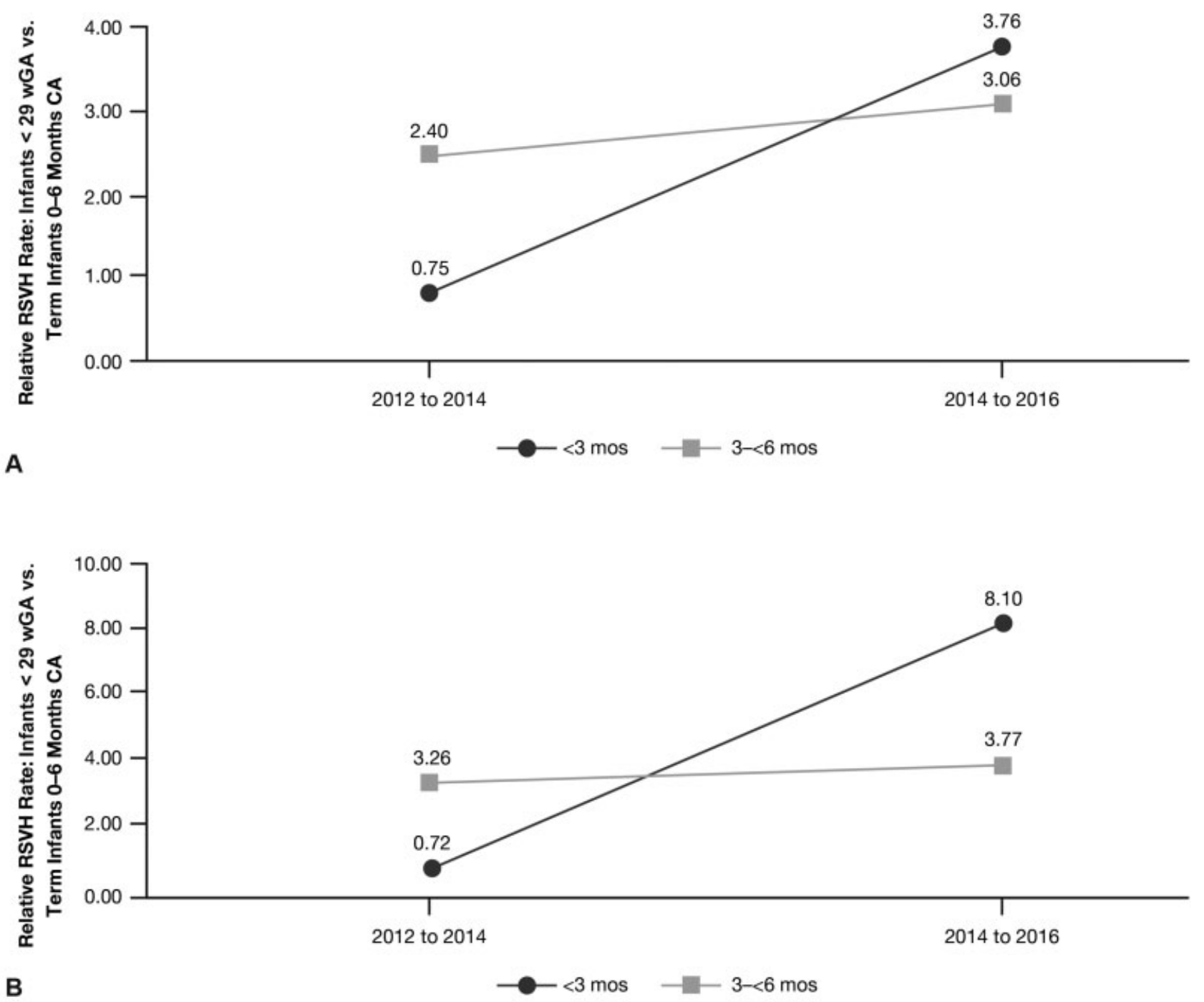

Fig. 2 RSV hospitalization rate ratios for infants $<29$ wGA versus term infants 0 to $<6$ months CA during the 2012 to 2014 and 2014 to 2016 RSV seasons. (A) Commercially insured infants; (B) Medicaid-insured infants. CA, chronological age; RSVH, respiratory syncytial virus hospitalization; wGA, weeks' gestational age.

Table 2 Change in respiratory syncytial virus and all-cause bronchiolitis hospitalization risks from 2012 to 2014 respiratory syncytial virus seasons to 2014 to 2016 respiratory syncytial virus seasons among infants <29 weeks' gestational age aged $<6$ months compared with term infants aged $<6$ months.

\begin{tabular}{|c|c|c|c|c|c|c|c|c|}
\hline & Commercial & & & & Medicaid & & & \\
\hline wGA & $\begin{array}{l}\text { Difference-in- } \\
\text { difference }^{\mathrm{a}} \\
\text { for RSV } \\
\text { hospitalization } \\
(95 \% \mathrm{Cl})\end{array}$ & $p$-value & $\begin{array}{l}\text { Difference-in- } \\
\text { difference for } \\
\text { all-cause } \\
\text { bronchiolitis } \\
\text { hospitalization } \\
(95 \% \mathrm{Cl})\end{array}$ & $p$-value & $\begin{array}{l}\text { Difference-in- } \\
\text { difference }^{\mathrm{a}} \\
\text { for RSV } \\
\text { hospitalization } \\
(95 \% \mathrm{Cl})\end{array}$ & $p$-value & $\begin{array}{l}\text { Difference-in- } \\
\text { difference for } \\
\text { all-cause } \\
\text { bronchiolitis } \\
\text { hospitalization } \\
(95 \% \mathrm{Cl})\end{array}$ & $p$-value \\
\hline$<29$ & $1.57(0.68-3.63)$ & 0.2867 & $0.64(0.32-1.25)$ & 0.1893 & $1.68(1.16-2.41)$ & 0.0054 & $1.53(1.16-2.02)$ & 0.0025 \\
\hline
\end{tabular}

Abbreviations: $\mathrm{Cl}$, confidence interval; RSV, respiratory syncytial virus; wGA, weeks' gestational age.

Note: Up to 6 months chronologic age.

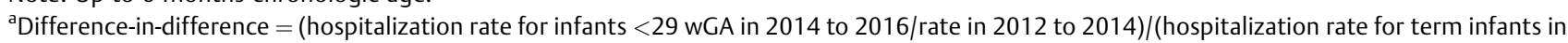
2014 to 2016/rate in 2012 to 2014).

exposure to RSV IP and hospitalization outcomes. Fourth, due to the small size of the $<29$ wGA population and resulting small study samples, the DID models had limited statistical power to detect a meaningful difference. Nevertheless, the Medicaid DID model demonstrated statistically significant increases in hospitalization risks, in part due to the larger number of infants in the Medicaid sample, and statistically significant declines in RSV IP receipt among Medicaid-insured infants 0 to $<3$ and 3 to $<6$ 
months CA. The DID model did not demonstrate a statistically significant difference in hospitalization risk in the Commercial population despite similar trends in the observed risks, presumably due to a smaller sample and the fact that the only significant decline in RSV IP receipt occurred among commercially insured infants $<3$ months CA. Lastly, although the largest impact on RSV IP receipt and RSVH risk occurred in infants $<3$ months $C A$, it was not possible to analyze infants 0 to $<3$ months CA in a separate DID model due to a small number of infant-seasons (Commercial: 121.7 from 2012 to 2014 and 87.9 from 2014 to 2016; Medicaid: 219.7 from 2012 to 2014 and 223.8 from 2014 to 2016) and associated small number of hospitalizations.

\section{Conclusion}

The 2014 change in the AAP policy on RSV IP use was associated with reduced outpatient RSV IP receipt and greater RSVH incidence among otherwise healthy infants $<29$ wGA, even though RSV IP continued to be recommended for this population. These findings warrant further study and attention due to the significant morbidity associated with severe RSV disease among preterm infants in the first months of life.

\section{Authors' Contributions}

All authors contributed to the study design, interpretation of results, and revisions of the manuscript. All authors had access to the full study analyses and have approved the final version of the manuscript. S.W.W. and A.M.K. contributed to the data analysis.

\section{Note}

L.B. and C.S.A. (employees of AstraZeneca) fulfilled all International Committee of Medical Journal Editors requirements for authorship of this manuscript and were therefore involved in study design; the collection, analysis, and interpretation of data; the writing of the manuscript; and the decision to submit the article for publication.

\section{Funding}

This study was supported by AstraZeneca (the manufacturer of palivizumab), which owned the U.S. rights to palivizumab at the time this work was completed. The U.S. rights to palivizumab are currently owned by Swedish Orphan Biovitrum AB (Sobi, Inc.).

\section{Conflict of Interest}

M.G. received grant/research support from AstraZeneca/ MedImmune and is a member of the AstraZeneca Speakers' Bureau. L.R.K. received grant and research support for clinical trials from AstraZeneca, Regeneron, Pfizer, and Sanofi Pasteur. He has also been a consultant to Pfizer. J.F. received grant/research support from AstraZeneca/MedImmune and is a member of the AstraZeneca and Sobi Speakers' Bureaus. L.B. and C.S.A. are employees of AstraZeneca. S.W.W. is a consultant to IBM Watson Health. A.M.K. is an employee of IBM Watson Health, which received funding from AstraZeneca to conduct this study.

\section{Acknowledgments}

The authors thank the editorial support provided by The Lockwood Group in accordance with Good Publication Practice (GPP3) and funded by AstraZeneca, and by ETHOS Health Communications in accordance with GPP3 and funded by Sobi, Inc.

\section{References}

1 Hall CB, Weinberg GA, Iwane MK, et al. The burden of respiratory syncytial virus infection in young children. N Engl J Med 2009;360 (06):588-598

2 Leader S, Kohlhase K. Recent trends in severe respiratory syncytial virus (RSV) among US infants, 1997 to 2000. J Pediatr 2003;143(5, Suppl):S127-S132

3 Boyce TG, Mellen BG, Mitchel EF Jr, Wright PF, Griffin MR. Rates of hospitalization for respiratory syncytial virus infection among children in Medicaid. J Pediatr 2000;137(06):865-870

4 Kong AM, Krilov LR, Fergie J, et al. The 2014-2015 national impact of the 2014 American Academy of Pediatrics guidance for respiratory syncytial virus immunoprophylaxis on preterm infants born in the United States. Am J Perinatol 2018;35(02):192-200

5 Goldstein M, Krilov LR, Fergie J, et al. Respiratory syncytial virus hospitalizations among US preterm infants compared to term infants before and after the 2014 American Academy of Pediatrics guidance on immunoprophylaxis: 2012-2016. Am J Perinatol 2018;35(14):1433-1442

6 Synagis ${ }^{\circledR}$ (palivizumab) [prescribing information]. Gaithersburg, MD: AstraZeneca Available at: https://www.azpicentral.com/ synagis/synagis.pdf\#page=1. Accessed: September 5, 2017

7 American Academy of Pediatrics. Respiratory syncytial virus. In: Pickering LK, Baker CJ, Kimberlin DW, Long SS, eds. Red Book: 2012 Report of the Committee on Infectious Diseases. 29th ed. Elk Grove Village: American Academy of Pediatrics; 2012:609-617

8 American Academy of Pediatrics Committee on Infectious Diseases; American Academy of Pediatrics Bronchiolitis Guidelines Committee. Updated guidance for palivizumab prophylaxis among infants and young children at increased risk of hospitalization for respiratory syncytial virus infection. Pediatrics 2014;134(02):415-420

9 Rajah B, Sánchez PJ, Garcia-Maurino C, Leber A, Ramilo O, Mejias A. Impact of the updated guidance for palivizumab prophylaxis against respiratory syncytial virus infection: a single center experience. J Pediatr 2017;181:183-188.e1

10 Ambrose CS. Is it RSV? Health Sci Rep 2018;1(12):e97

11 Turi KN, Wu P, Escobar GJ, et al. Prevalence of infant bronchiolitiscoded healthcare encounters attributable to RSV. Health Sci Rep 2018;1(12):e91

12 Makari D, Staat MA, Henrickson KJ, Wu X, Ambrose CS. The underrecognized burden of respiratory syncytial virus among infants presenting to US emergency departments. Clin Pediatr (Phila) 2015;54(06):594-597

13 Ralston SL, Lieberthal AS, Meissner HC, et al; American Academy of Pediatrics. Clinical practice guideline: the diagnosis, management, and prevention of bronchiolitis. Pediatrics 2014;134(05):e1474-e1502

14 Bardach NS, Cabana MD. The unintended consequences of quality improvement. Curr Opin Pediatr 2009;21(06):777-782

15 Centers for Medicare \& Medicaid. Measures Management System. February 2018. Available at: https://www.cms.gov/Medicare/ Quality-Initiatives-Patient-Assessment-Instruments/MMS/Downloads/Clinical-Guidelines.pdf. Accessed December 26, 2018

16 Ursu A, Sen A, Ruffin M. Impact of cervical cancer screening guidelines on screening for chlamydia. Ann Fam Med 2015;13(04):361-363

17 Pletcher MJ, Fernandez A, May TA, et al. Unintended consequences of a quality improvement program designed to improve treatment of alcohol withdrawal in hospitalized patients. Jt Comm J Qual Patient Saf 2005;31(03):148-157 